\title{
2 EFICÁCIA DO PROGRAMA INCARE NA SOBRECARGA DOS CUIDADORES INFORMAIS DE PESSOAS IDOSAS APÓS UM AVC
}

\author{
| Odete Araújo ${ }^{1}$; Isabel Lage ${ }^{2}$; José Cabrita ${ }^{3}$; Laetitia Teixeira ${ }^{4} \mid$
}

\section{RESUMO}

CONTEXTO: Em Portugal o acidente vascular cerebral (AVC) representa a principal causa de incapacidade nas pessoas idosas. Apesar dos avanços nos cuidados de saúde permitirem que mais sobreviventes de AVC vivam no domicílio cuidados pelas famílias, a evidência sugere que os cuidadores sofrem elevados níveis de sobrecarga e insatisfação com a quantidade e qualidade da informação e com o suporte recebido. O estado da arte sugere, ainda, a necessidade da implementação de programas de intervenção psicoeducativa baseados na resolução de problemas e na aquisição de competências práticas, visando a redução do impacte dos cuidados nos cuidadores.

OBJETIVO(S): Desenvolveu-se um estudo tendo como objetivos: (1) avaliar as competências práticas dos cuidadores de pessoas idosas sobreviventes de um AVC; e (2) avaliar um programa de intervenção na diminuição de sobrecarga destes cuidadores no $1^{\circ}$ (M1) e $3^{\circ}$ (M2) mês após a intervenção.

METODOLOGIA: O estudo quasi-experimental foi realizado com uma amostra de 174 cuidadores informais de pessoas idosas dependentes sobreviventes de AVC, os quais foram distribuídos pelo grupo experimental $(n=85)$ e pelo grupo controlo ( $n=89)$. A investigação decorreu nos domicílios de famílias inscritas em Agrupamentos de Centros de Saúde da região norte.

RESULTADOS: Os dados sugerem elevados níveis de sobrecarga no $1^{\circ}$ e $3^{\circ}$ mês no grupo de controlo, comparativamente com o grupo experimental, no qual se verificou uma diminuição após o follow-up (M2).

CONCLUSÃO: Pode concluir-se que os resultados da implementação do programa InCARE corroboram a importância de uma intervenção precoce e estruturada na capacitação e redução da sobrecarga dos cuidadores.

PALAVRAS-CHAVE: Cuidadores informais; Pessoas idosas; AVC; Estudo quasi-experimental

\section{RESUMEN}

"Eficacia del programa InCARE en la sobrecarga de los cuidadores informales de personas ancianas después de un AVC"

CONTEXTO: En Portugal el accidente vascular cerebral (AVC) representa la principal causa de incapacidad en la personas de edad. A pesar de los avances en los cuidados de salud permitir que más supervivientes de AVC vivan en el domicilio al cuidado de sus familias, la evidencia sugiere, todavía, que los cuidadores sufren elevados niveles de sobrecarga e insatisfacción con la cantidad y calidad de la información y con el apoyo recibido. El estado del arte traduce la necesidad de la implementación de programas de intervención psicoeducativa basados en la resolución de problemas y en la adquisición de competencias prácticas como condicionantes de la reducción del impacto de os cuidados de la salud y sobrecarga de los cuidadores.

OBJETIVO(S): Fue desarrollado un estudio que tuvo como objetivos: (1) evaluar las competencias prácticas de los cuidadores de personas ancianas supervivientes de un AVC; (2) evaluar un programa de intervención en la disminución de sobrecarga de estos cuidadores en el $1^{\circ}$ (M1) y $3^{\circ}$ (M2) mes después de la intervención.

METODOLOGÍA: Estudio casi-experimental realizado com una muestra de 174 cuidadores informales de personas ancianas dependientes supervivientes de AVC, distribuidos por el grupo experimental $(n=85)$ y por el grupo de control $(n=89)$. La investigación transcurrió en los domicilios de familias inscritas en Agrupaciones de Centros de Salud de la región norte de Portugal.

RESULTADOS: Los datos sugieren elevados niveles de sobrecarga en el grupo de control en el $1^{\circ}$ y el $3^{\circ}$ mes, comparativamente con el grupo experimental, en el cual se verificó una disminución después del follow-up (M2).

CONCLUSIÓN: Los resultados de la implementación del programa InCARE corroboran la importancia de una intervencione precoce y estructurada en la capacitación y reducción de la sobrecarga.

\section{DESCRIPTORES: Cuidadores informales; Personas ancianas; AVC; Estudio casi-experimental}

\section{ABSTRACT \\ "Efficacy of the program InCARE in the burden of informal caregivers of older people after a stroke"}

BACKGROUND: Stroke is the leading cause of disability in Portuguese older people. Despite the advances in the health care system, allowing more stroke survivors living at home be cared by their families, the evidence suggests higher levels of burden in caregivers, dissatisfaction with the quantity and quality of information they received and with the support received. The state-of-the art, also suggests, there is a need for implementing a psychoeducational intervention programmes based on problem solving and on practical skills acquisition to reduce burden in caregivers.

AIM: The study aimed at: (1) assessing the practical skills of caregivers of older stroke survivors; (2) evaluating an intervention program to reduce burden in caregivers during the 1st (M1) and 3rd (M2) month after the intervention.

METHODOS: The quasi-experimental study was developed with 174 informal caregivers of dependent older stroke survivors, allocated in: i) experimental group $(\mathrm{n}=85)$; and ii) control group $(\mathrm{n}=89)$. Data collection occurred in community-dwelling of health care units in northern Portugal.

RESULTS: The data suggests higher levels of burden in the 1st and 3rd month of the control group in comparison with the experimental group, in which a decrease after a follow-up was verified. (M2).

CONCLUSION: The results of the InCARE program suggests the importance of early structured interventions to achieve practical skills by informal caregivers of older stroke survivors as a therapeutic intervention to reduce burden.

\section{KEYWORDS: Caregivers; Older people; Stroke; Quasi-experi- mental design}

Submetido em 13-11-2015

Aceite em 19-02-2016

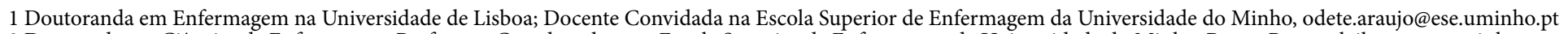

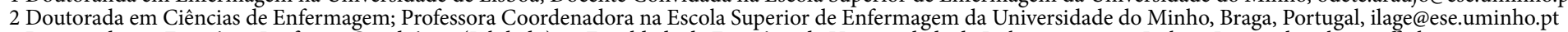

3 Doutorado em Farmácia; Professor Catedrático (Jubilado) na Faculdade de Farmácia da Universidade de Lisboa, 1649-003 Lisboa, Portugal, jcabrita@ff.ul.pt

4 Doutorada em Matemática; Professora Auxiliar Convidada no Instituto de Ciências Biomédicas Abel Salazar, 4050-313 Porto, Portugal, lcteixeira@icbas.up.pt

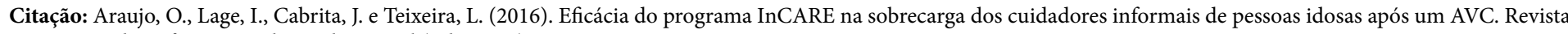
Portuguesa de Enfermagem de Saúde Mental (Ed. Esp. 3), 09-13. 


\section{INTRODUÇÃO}

Nos países desenvolvidos, o acidente vascular cerebral (AVC) representa a primeira causa de morbilidade geradora de dependência (Feigin et al., 2014; Truelsen et al., 2006). Em Portugal existem 97,6 / 100,000 mortes por ano (Correia et al., 2004). De acordo com Mendonça, Machado e Teixeira (2009) mais de $42,5 \%$ dos portugueses com mais de 75 anos de idade sofrem uma doença cerebrovascular e a maioria dos sobreviventes necessita de ajuda nas atividades do autocuidado, após a alta hospitalar.

Apesar dos avanços nos cuidados de saúde, permitindo que mais sobreviventes de AVC vivam no domicílio cuidados pelas famílias, a evidência sugere que os cuidadores reportam insatisfação com a quantidade, qualidade da informação e do suporte recebido, potenciando elevados níveis de sobrecarga nos cuidadores (Shyu, Kuo, Chen, \& Chen, 2010).

A meta-análise realizada por Legg e colaboradores (Legg et al., 2012) e publicada na Cochrane, sobre intervenções não farmacológicas dirigidas a cuidadores informais de sobreviventes de AVC, dá conta da importância da implementação de intervenções psico-educativas baseadas na informação e no treino dos cuidadores informais como estratégias eficazes na redução da sobrecarga e da ansiedade destes prestadores de cuidados.

Por sua vez, os resultados do estudo de Cheng e colaboradores (2014) vêm dizer que através do treino de competências dos cuidadores, é possível obter uma redução nos custos com a saúde. Também as diretrizes do National Institute for Care and Health Excellence sugerem o envolvimento efetivo das famílias no processo de tomada de decisão na gestão dos cuidados aos sobreviventes de AVC, bem como, uma intervenção sistemática na aquisição de conhecimentos e competências práticas, no sentido de evitar readmissões hospitalares ou a institucionalização precoce dos sobreviventes de AVC (NICE, 2013).

Atualmente, em Portugal, não são conhecidos programas de intervenção estruturada que promovam competências práticas nos cuidadores informais de idosos dependentes após um AVC, com avaliação do impacte na diminuição da sobrecarga. Neste sentido, desenvolveu-se um estudo tendo como objetivos: (1) avaliar as competências práticas dos cuidadores de pessoas idosas sobreviventes de um AVC; e (2) avaliar um programa de intervenção na diminuição de sobrecarga destes cuidadores no $1^{\circ}$ (M1) e $3^{\circ}$ (M2) mês após a intervenção.

\section{METODOLOGIA}

Adotou-se um desenho quasi-experimental (Portney \& Watkins, 2009), com recurso a uma amostra de 174 cuidadores informais de pessoas idosas dependentes sobreviventes de AVC, os quais foram distribuídos pelo grupo experimental $(\mathrm{n}=85)$ e pelo grupo controlo ( $\mathrm{n}=89$ ) (Figura 1). A investigação decorreu nos domicílios de famílias cuidadoras inscritas em Agrupamentos de Centros de Saúde da região norte.

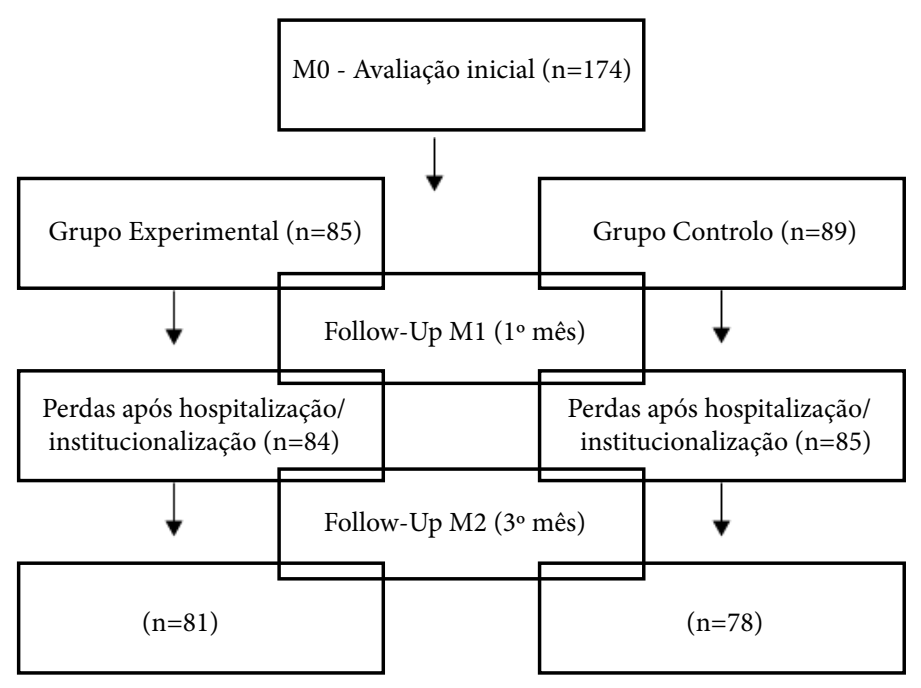

Figura 1 - Diagrama do fluxo dos participantes ao longo do estudo

\section{Instrumentos de Recolha de Dados}

- Escala de Capacidades do Prestador Informal de Cuidados de Idosos Dependentes após um AVC (ECPICID-AVC) (Araújo, Lage, Cabrita e Teixeira, 2015).

- Questionário de Avaliação da Sobrecarga para Cuidadores Informais (QASCI) (Martins, Ribeiro e Garrett, 2003).

\section{Intervenção}

Avaliação das competências práticas dos cuidadores informais na $1^{\text {a }}$ semana, $1^{\circ}$ e $3^{\circ}$ mês após o regresso a casa; avaliação da sobrecarga ao $1^{\circ}$ (M1) e $3^{\circ}$ (M2) mês após a intervenção.

\section{Análise dos Dados}

Todas as análises foram efetuadas com recurso ao software SPSS ${ }^{\circledR} 22.0$ (SPSS ${ }^{\circledR}$ Inc., Chicago, IL, USA), e em todas as análises foi considerado o nível de significância de 0.05 . De modo a identificar potenciais variáveis diferenciadoras dos grupos em estudo (grupo experimental e grupo de controlo), foi realizada uma análise bivariada entre a variável grupo e todas as variáveis consideradas na análise descritiva. 
A avaliação do impacto da intervenção foi avaliada através da evolução dos seguintes indicadores: capacitação do cuidador informal - ECPICID-AVC e sobrecarga do cuidador - QASCI. A análise longitudinal do ECPICID-AVC e do QASCI foi efetuada com recurso aos modelos lineares mistos.

\section{Considerações Éticas}

A investigação realizada teve o parecer favorável (parecer n44/2013) da Comissão de Ética da Administração Regional de Saúde do Norte em 28 de maio de 2013. Todos os procedimentos foram levados a cabo tendo em consideração o consentimento informado dos participantes no que concerne à participação em investigação de acordo com a declaração de Helsínquia e a convenção de Oviedo. Foi explicado aos cuidadores que a participação seria voluntária, sem custos para as famílias, e que todos os dados seriam tratados de forma anónima e confidencial. A implementação do programa InCARE foi registado no clinicaltrials.gov com o ID number NCT02074501.

\section{RESULTADOS}

\section{Caraterização da Amostra}

A amostra de cuidadores informais é maioritariamente constituída por cuidadores do sexo feminino (155, 89,1\%). A idade mínima é de 29 anos e a idade máxima de 82 , sendo a média e o desvio padrão das idades de 55,2 anos e 11,3 anos respetivamente. Cento e vinte e um cuidadores $(69,5 \%)$ são casados, $28(16,1 \%)$ solteiros, $22(12,6 \%)$ divorciados e apenas $3(1,7 \%)$ viúvos. Analisando o nível educacional, 22 cuidadores informais $(12,6 \%)$ apenas sabem ler e escrever, mais de metade dos cuidadores que integram o estudo apresenta entre 1 a 4 anos de escolaridade $(93,53,4 \%), 42(24,1 \%)$ entre $5-9$ anos de escolaridade, $10(5,7 \%)$ entre 10 e 12 anos e, finalmente 7 cuidadores $(4,0 \%)$ possuem mais de 12 anos de escolaridade. No que diz respeito à situação profissional, 29 (16,7\%) dos cuidadores estão empregados, 54 (31,0\%) estão desempregados, $42(24,1 \%)$ são domésticos e 49 (28,2\%) são reformados. $\mathrm{O}$ escalão de rendimento mais mencionado variou entre 500 e $1000 €(124,71,3 \%)$, seguindo-se o escalão $250-500 €(34,19,5 \%),>1000 €(13,7,5 \%)$ e por fim o $<250 €(3,1,7 \%)$. Os dados relativos à caracterização sociodemográfica dos cuidadores que integram este estudo são apresentados na tabela 1 .
Tabela 1 - Perfil biográfico e social dos cuidadores informais

\begin{tabular}{|c|c|c|c|c|c|}
\hline & \multicolumn{4}{|c|}{ Grupo } & \multirow{3}{*}{$\mathrm{p}$} \\
\hline & \multicolumn{2}{|c|}{ Controlo } & \multicolumn{2}{|c|}{ Experimental } & \\
\hline & $\mathrm{n}$ & $\%$ & $\mathrm{n}$ & $\%$ & \\
\hline $\begin{array}{l}\text { Género } \\
\text { Feminino } \\
\text { Masculino }\end{array}$ & $\begin{array}{c}80 \\
9\end{array}$ & $\begin{array}{l}51,6 \\
47,4\end{array}$ & $\begin{array}{l}75 \\
10\end{array}$ & $\begin{array}{l}48,4 \\
52,6\end{array}$ & 0,727 \\
\hline Idade, média (dp) & \multicolumn{2}{|c|}{$56,0(11,2)$} & \multicolumn{2}{|c|}{$54,3(11,5)$} & 0,348 \\
\hline $\begin{array}{l}\text { Estado civil } \\
\text { Solteiro } \\
\text { Casado } \\
\text { Viúvo } \\
\text { Divorciado }\end{array}$ & $\begin{array}{c}18 \\
58 \\
2 \\
11\end{array}$ & $\begin{array}{l}64,3 \\
47,9 \\
66,7 \\
50,0\end{array}$ & $\begin{array}{c}10 \\
63 \\
1 \\
11\end{array}$ & $\begin{array}{l}35,7 \\
52,1 \\
33,3 \\
50,0\end{array}$ & $0,454^{\star}$ \\
\hline $\begin{array}{l}\text { Educação } \\
\text { Sabe ler/escrever } \\
1-4 \text { anos } \\
5-9 \text { anos } \\
10-12 \text { - anos } \\
>12 \text { anos }\end{array}$ & $\begin{array}{c}10 \\
52 \\
18 \\
6 \\
3\end{array}$ & $\begin{array}{l}45,5 \\
55,9 \\
42,9 \\
60,0 \\
42,9\end{array}$ & $\begin{array}{c}12 \\
41 \\
24 \\
4 \\
4\end{array}$ & $\begin{array}{l}54,5 \\
44,1 \\
57,1 \\
40,0 \\
57,1\end{array}$ & $0,586^{*}$ \\
\hline $\begin{array}{l}\text { Situação profissional } \\
\text { Empregado } \\
\text { Desempregado } \\
\text { Doméstico } \\
\text { Reformado }\end{array}$ & $\begin{array}{l}16 \\
24 \\
19 \\
30\end{array}$ & $\begin{array}{l}55,2 \\
44,4 \\
45,2 \\
61,2\end{array}$ & $\begin{array}{l}13 \\
30 \\
23 \\
19\end{array}$ & $\begin{array}{l}44,8 \\
55,6 \\
54,8 \\
38,8\end{array}$ & 0,291 \\
\hline
\end{tabular}

*teste exato de Fisher

Dos 174 cuidadores informais que participaram neste estudo, $89(51,1 \%)$ foram alocados ao grupo de controlo e $85(48,9 \%)$ ao grupo experimental. Através da análise dos resultados obtidos, verifica-se uma interação estatisticamente significativa entre o tempo e o grupo $(\mathrm{p}<0,001)$. Existe um efeito diferenciado do programa no score médio do QASCI de acordo com o grupo. Através da análise da tabela 2, pode verificarse que, mesmo após ajustar para as variáveis idade do cuidador $(\mathrm{p}=0,299)$, número de horas de cuidado $(\mathrm{p}=0,354)$, a existência de outro cuidador $(\mathrm{p}=0,696)$ e a proveniência de uma UCC $(\mathrm{p}=0,236)$, verifica-se que o grupo experimental apresenta uma diminuição da sobrecarga, contrariamente ao grupo controlo que apresenta um aumento. A tabela 2 apresenta os resultados da sobrecarga do cuidador informal (QASCI) com base no Modelo Linear Misto.

Tabela 2 - Modelo Linear Misto para o QASCI

\begin{tabular}{|l|c|c|c|c|}
\hline \multicolumn{1}{|c|}{ Variável } & Estimativa & Erro padrão & $\mathbf{p}$ & IC 95\% \\
\hline $\begin{array}{l}\text { Tempo } \\
\text { M1 }\end{array}$ & $-6,36$ & 1,87 & 0,001 & $-10,0--2,67$ \\
M2 (ref) & - & - & - & - \\
\hline $\begin{array}{l}\text { Grupo } \\
\text { Experimental } \\
\text { Controlo (ref) }\end{array}$ & $-15,1$ & 2,28 & $<0,001$ & $-19,6--10,7$ \\
\hline $\begin{array}{l}\text { Interação } \\
\text { M1* Experi- } \\
\text { mental }\end{array}$ & 10,7 & 2,66 & $<0,001$ & $5,45-15,9$ \\
\hline
\end{tabular}




\section{DISCUSSÃO}

Este estudo procurou avaliar a eficácia de um programa de intervenção (InCARE) assente na capacitação dos cuidadores informais de pessoas idosas dependentes sobreviventes de AVC e reduzir a sobrecarga deste grupo de pessoas.

Os dados sugerem elevados níveis de sobrecarga no $1^{\circ}$ e $3^{\circ}$ mês no grupo de controlo, comparativamente com o grupo experimental, no qual se verificou uma diminuição após o follow-up (M2) (Cheng, Chair \& Pak-Chun, 2014; Clarke, 2014; Forster et al., 2013; Hafsteinsdóttir, Vergunst, Lindeman \& Schuurmans, 2011; Pandian, 2015; Shyu et al., 2010), vindo também ao encontro do subscrito por Legg e colaboradores (2012), quando reforçam a importância de uma intervenção psicoeducativa baseada na informação e no treino, como estratégias eficazes na redução da sobrecarga dos cuidadores informais.

Pode dizer-se ainda que os resultados da implementação do programa InCARE encontram expressão em Kalra et al, (2004); Smith, Forster \& Young (2004); Gräsel et al, (2005); Hartke \& King (2003); Marsden et al, (2010); Torp et al, (2008), bem como nos de Perrin e colaboradores (2010), uns e outro, unânimes ao considerarem a intervenção estruturada sobre conhecimento e treino, como uma forma de aliviar os efeitos dos cuidados na saúde e bem-estar dos cuidadores informais de pessoas dependentes.

\section{CONCLUSÃO}

Os resultados deste estudo plasmam a importância da intervenção precoce e estruturada baseada na informação, no treino e na capacitação dos cuidadores informais de sobreviventes de AVC, como forma de diminuir a sobrecarga e promover a saúde física, psicológica, o bem-estar social e a satisfação com os cuidados.

\section{IMPLICAÇÕES PARA A PRÁTICA CLÍNICA}

Os enfermeiros como figura de referência e agentes promotores de saúde e bem-estar de doentes e famílias em geral, e em particular dos sobreviventes de AVC e dos seus cuidadores informais, devem privilegiar a intervenção precoce e estruturada com enfoque na capacitação (informação e treino) e na redução da sobrecarga destes prestadores de cuidados.

\section{REFERÊNCIAS BIBLIOGRÁFICAS}

Araújo, O., Lage, I., Cabrita, J., e Teixeira, L. (2015). Development and psychometric properties of ECPICID-AVC to measure informal caregivers' skills when caring for older stroke survivors at home. Scandinavian Journal of Caring Sciences, in press.

Cheng, H. Y., Chair, S. Y., \& Chau, J. P. (2014). The effectiveness of psychosocial interventions for stroke family caregivers and stroke survivors: A systematic review and meta-analysis. Patient Education and Counseling, 95(1), 30-44. doi: 10.1016/j. pec.2014.01.005

Clarke, D. H., Sadler, E., Harding, G., McKevitt, G., Godfrey, M., Dickerson, J., Farrin, A.J., Kalra, L., Smithard, D. \& Forster, A. (2014). Introducing structured caregiver training in stroke care: findings from the TRACS process evaluation study. BMJ Open, 4, 1-10. doi: 10.1136/bmjopen-2013-004473

Correia, M., Silva, M., Matos, I., Magalhães, R., Lopes, J., Ferro, J., e Silva, C. (2004). Prospective community-based study of stroke in Northern Portugal: incidence and case fatality in rural and urban populations. Stroke: Journal of the American Heart Association, 35, 2048-2053.

Draper, B., Bowring, G., Thompson, C., Heyst, J. V., Conroy, P., \& Thompson, J. (2007). Stress in caregivers of aphasic stroke patients: a randomized controlled trial. Clinical Rehabilitation, 21(2), 122-130.

Feigin, V., Forouzanfar, M., Krishnamurthi, R., Mensah, G., Connor, M., \& Bennett, D. (2014). Global and regional burden of stroke during 1990-2010: findings from the Global Burden of Disease Study. Lancet, 18(383), 245-254.

Forster, A., Dickerson, J., Young, J., Patel, L., Nixon, J., Smithard, D., ... Farrin, A. (2013). A structured training for caregivers of inpatients after stroke (TRACS): a cluster randomised controlled trial and cost-effectiveness analysis. Lancet, 382(9910), 2069-2076. 
Gräsel, E., Biehler, J., Schmidt, R., \& Scupp, W. (2005). Intensification of the transition between inpatient neurological rehabilitation and home care of stroke patients. Controlled trial with follow-up assessment six months after discharge. Clinical Rehabilitation, 19(7), 725-736.

Hafsteinsdóttir, T., Vergunst, M., Lindeman, E., \& Schuurmans, M. (2011). Educational needs of patients with a stroke and their caregivers: a systematic review of the literature. Patient Education and Counseling, 85(1), 14-25.

Hartke, R. J., \& King, R. B. (2003). Telephone group intervention for older stroke survivors. Top Stroke Rehabilitation, 9(4), 65-81.

Kalra, L., Evans, A., Perez, I., Melbourn, A., Patel, A., Knapp, M., \& Donaldson, N. (2004). Training carers of stroke patients: randomised controlled trial. BMJ, 328, 1-5.

Legg, L., Quinn, T., Mahmood, F., Weir, C., Tierney, J., Stott, D., ... Langhorne, P. (2012). Nonpharmacological interventions for caregivers of stroke survivors. Stroke: Journal of the American Heart Association, 43, 30-31. doi: 10.1161/STROKEAHA.111.644914

Lincoln, N., Walker, M., Dixon, A., \& Knights, P. (2004). Evaluation of a multiprofessional community stroke team: a randomized controlled trial. Clinical Rehabilitation, 18(1), 40-47.

Marsden, D., Quinn, R., Pond, N., Golledge, R., Neilson, C., White, J., ... Ollack, M. (2010). A multidisciplinary group in rural settins for community-dwelling chronic stroke survivors and their carers: a pilot randomized controlled trial. Clinical Rehabilitation, 24(4), 328-341.

Martins, T., Ribeiro, J., e Garrett, C. (2003). Estudo de validação do Questionário de Avaliação da Sobrecarga para Cuidadores Informais. Psicologia, Saúde \& Doenças, 4(1), 131-148.

Mendonça, M., Machado, F., e Teixeira, C. (2009). Perfil de saúde da região Norte. Acedido em: http:// portal.arsnorte.min-saude.pt/portal/page/portal/ ARSNorte/Conteúdos/Saúde Pública Conteudos/ Perfil_RN_2009.pdf
National Institue for Health and Care Excellence. (2013). Stroke rehabilitation Long-term rehabilitation after stroke. Manchester, United Kingdom: NICE.

Pandian, J. D. F., Kaur, P., Sharma, D., Julia, L., Toor, G., Arora, R., ... Lindley, R. (2015). Family-Led rehabilitation after Stroke in india: the ATTEND pilot study. International Journal of Stroke, 10(4), 609-614. doi: $10.1111 /$ ijs.12475

Perrin, P. B., Johnston, A., Vogel, B., Heesacker, M., Veja-Trujillo, M., Anderson, J. \& Rittman, M. (2010). A culturally sensitive transition assistance program for stroke caregivers: examining caregiver mental health and stroke rehabilitation. Journal of Rehabilitation Research and Development, 47(7), 605-616.

Portney, L. G., \& Watkins, M. P. (2009). Foundations of Clinical Research: applications to practice. New Jersey, United States of America: Pearson International Edition.

Shyu, Y.-I. L., Kuo, L.-M., Chen, M.-C., \& Chen, S.T. (2010). A clinical trial of an individualised intervention programme for family caregivers of older stroke victims in Taiwan. Journal of Clinical Nursing, 19 (11-12), 1675-1685. doi: 10.1111/j.13652702.2009.03124.x.

Smith, J., Forster, A., \& Young, J. (2004). A randomized trial to evaluate an education programme for patients and carers after a stroke. Clinical Rehabilitation, 18(7), 726-736.

Torp, S., Hanson, E., Hauge, S., Ulstein, I., \& Magnusson, L. (2008). A pilot study of how information and communication technology may contribute to health promotion among elderly spousal carers in Norway. Health Social Care Community 16(1), 75-85. doi: 10.1111/j.1365-2524.2007.00725.x.

Truelsen, T., Piechowski-Józwiak, B., Bonita, R., Mathers, C., Bogousslavsky, J., \& Boysen, G. (2006). Stroke incidence and prevalence in Europe: a review of available data. European Journal of Neurology, 13(6), 581-598. 